\title{
Factores asociados al uso de tecnologías de la información y la comunicación (TIC) en los procesos de aprendizaje de estudiantes de ingeniería
}

\author{
Davinson Mosquera-González ${ }^{1}$, Alejandro Valencia-Arias ${ }^{2,3 *}$, Martha Benjumea-Arias ${ }^{4} y$ \\ Lucia Palacios-Moya ${ }^{1}$ \\ (1) Centro de Investigaciones de Escolme (CIES). Institución Universitaria Escolme. Cl. 50 \#40-39 Medellín - Colombia \\ (correo-e: dmosquerag@escolme.edu.co; cies@escolme.edu.co) \\ (2) Facultad de Ingeniería, Corporación Universitaria Americana, Carrera 43 \# 49-57, Medellín, Colombia. \\ (correo-e: correo-e: javalenciar@gmail.com) \\ (3) Instituto de Investigación, Universidad Católica Los Ángeles de Chimbote, Jirón Tumbes 247, Chimbote 02804, \\ Chimbote - Perú. (correo-e: javalenciar@gmail.com). \\ (4) Facultad de Ciencias Económicas y Administrativas, Departamento de Finanzas, Instituto Tecnológico Metropolitano \\ - ITM, Calle 54 A \#30-01, Medellín - Colombia (correo-e: marthabenjumea@itm.edu.co). \\ $\left({ }^{*}\right)$ Autor a quien debe dirigirse la correspondencia
}

Recibido Jul. 24, 2020; Aceptado Sep. 22, 2020; Versión final Oct. 20, 2020, Publicado Abr. 2021

\begin{abstract}
Resumen
El objetivo de la presente investigación fue examinar los factores asociados al uso de tecnologías de la información y la comunicación (TIC) en procesos de aprendizaje en estudiantes universitarios. Se realizó un estudio cuantitativo de campo a través de la aplicación de encuestas a estudiantes inscritos en programas de ingeniería industrial y administrativa de la Universidad Nacional (Medellín, Colombia). Se identificaron nueve componentes como soporte del modelo propuesto. Se encontró una asociación significativa entre el reconocimiento de la importancia del uso de software como soporte en la formación y la utilidad de uso percibido de las TIC; así como una relación significativa entre el reconocimiento de importancia del uso de las TIC y la percepción de autoeficacia. Los hallazgos de este estudio arrojan evidencia sobre la correlación que existe entre las habilidades y capacidades para el uso de TIC y la autoeficacia percibida de los estudiantes que condiciona su empleo en contextos educativos y de formación profesional.
\end{abstract}

Palabras clave: tecnologías de la información; estudiantes universitarios; educación mediada; superior; enseñanza

\section{Factors associated with using information and communication technologies (ICT) in the learning processes of engineering students}

\begin{abstract}
The aim of this research study was to examine factors associated with the use of information and communication technologies (ICT) and university student learning processes. A quantitative field study was performed by surveying students in industrial and administrative engineering programs at the National University (Medellín, Colombia). Nine components were identified and used to propose a model. The model aimed at evaluating the willingness to use ICTs as support for professional training of engineering students. The results showed that there was a significant association between acknowledging the importance of using software as support for training and perceived utility of using ICTs. There was also a significant correlation between recognizing the importance of using ICTs and self-efficacy perception. It is concluded that there was evidence to support a correlation between skills and abilities for using ICTs and student perception of selfefficacy, which determines the applications of ICTs in educational and professional training.
\end{abstract}




\section{INTRODUCCIÓN}

El papel de las Tecnologías de la Información y Comunicación -TIC- en la enseñanza y el aprendizaje, se ha convertido en uno de los temas más importantes de debate en los programas actuales de educación universitaria, haciendo parte fundamental en el desarrollo del sistema educativo (Gasaymeh, 2018). Estas tecnologías han cambiado las estructuras sociales, el estilo de vida, los procesos comunicativos e incluso la manera de educar, generando la llamada sociedad de la comunicación y del conocimiento (Maleki et al., 2012). Lo que da paso a una "sociedad del conocimiento", la cual se basa en una fuerza de trabajo con habilidades de alto nivel en el manejo de herramientas TIC (Guerin et al., 2017).

En este sentido, las Instituciones de Educación Superior -IES- tienen un rol clave en la promoción del conocimiento y la innovación, pero no puede desconocerse que este nuevo escenario es un desafío inesperado difícil de enfrentar. Por tanto, incorporar la enseñanza de las TIC dentro de los planes de estudio en las universidades, como un tema de capacitación cruzada, es un desafío complejo pero necesario en la preparación de los estudiantes para el éxito en el mercado laboral (Picatoste et al., 2018). Según los estudios de Cobo (2009), las TIC se definen como "dispositivos tecnológicos" (hardware y software), que permiten editar, producir, almacenar, intercambiar y transmitir datos entre diferentes sistemas de información que tienen protocolos comunes, cuyas características más significativas giran en torno a sus capacidades tecnológicas, las cuales son cada vez mayores con costos en disminución constante; además, el avance en éstas se ha convertido en una propuesta de educación que utiliza y promueve una estrategia de formación más autónoma por parte del estudiante, cercana y pertinente respecto a una aproximación individual y grupal, en la construcción de conocimiento (Meléndez y Flores, 2017).

Por ello, cuando se considera que la educación durante los últimos siglos ha estado usando la tecnología para expandir y desarrollar diferentes procesos, no es sorprendente que la llegada de las TIC esté siendo usada para obtener conocimientos a través de nuevos métodos (Hamidi et al., 2011). Es así como la educación ha dado un salto, por ejemplo, en el aprendizaje virtual, el cual es un proceso que utiliza medios de comunicación o equipos electrónicos (herramientas TIC) como ayuda, donde se espera que mejore la efectividad y la eficiencia del aprendizaje (Soeparno y Muslim, 2018). Por eso, se ha dicho que las formas de integrar e implementar las TIC en procesos educativos, se han ido transformando, implicando esfuerzos menores y un acceso más libre a contenidos y estrategias de aprendizaje según el contexto, en esa medida se ha querido que para que se desarrollen estas actividades educativas cuenten con los "requisitos y estándares internacionales de calidad que garanticen su usabilidad técnica" (Martínez, et a., 2018). Dado lo anterior, la implementación y utilización de las TIC en el proceso de enseñanza y aprendizaje tiene múltiples beneficios, tales como acelerar y facilitar algunos procesos de aprendizaje, el acceso a información nueva e interesante para el estudiante, posibilidad de procesos de apropiación del conocimiento más fáciles que los tradicionales en donde no hay uso de TIC, además de eliminar limitaciones de espacio y tiempo; permitiendo al estudiante adquirir conceptos de forma práctica y creativa (Bidarian et al., 2011).

Así, con el contexto descrito, surge la importancia de estudios que profundicen en el entendimiento de variables asociadas al uso de las TIC en relación con procesos de enseñanza y aprendizaje, teniendo en cuenta las regiones de análisis. Es el caso de esta investigación, que busca indagar sobre los factores que inciden en el uso de TIC en los procesos de aprendizaje en estudiantes de ingeniería administrativa e industrial de la Universidad Nacional de Medellín, Colombia. Para lograrlo es indispensable presentar una conceptualización de lo que se entiende por autoeficacia, ya que esta es la manera en la que un individuo identifica sus capacidades y actúa de acuerdo con estas para alcanzar un rendimiento deseado, es decir, es la aptitud para manejar y superar diversas situaciones complejas (Ornelas, et al., 2012). La autoeficacia transversaliza el uso e implementación de las TIC, ya que orienta, desde la percepción y análisis de contextos, cómo alcanzar de manera satisfactoria un objetivo.

\section{METODOLOGÍA}

Para el desarrollo de esta investigación se utilizó un enfoque metodológico cuantitativo de diseño no experimental y corte transversal de campo, de alcance correlacional; en el que se midió el grado de asociación entre las variables y componentes de análisis, representadas a partir de 12 hipótesis de estudio. Para detallar al lector la ruta metodológica que orientó la investigación, se presentan a continuación la composición de la muestra referencial de los participantes del estudio, las hipótesis formuladas, así como las técnicas para la recolección -procedimiento- y análisis de datos.

\section{Muestra}

La muestra participante en esta investigación estuvo compuesta por un total de 115 estudiantes de ingeniería industrial y administrativa de la Universidad Nacional de Colombia -Sede Medellín-, elegidos por un muestreo 
no probabilístico por criterio; de los cuales el 55\% pertenecen al programa de ingeniería industrial y el $45 \%$ a ingeniería administrativa; además, respecto al semestre cursado, pudo identificarse que el $39 \%$ y $49 \%$ de los estudiantes encuestados cursaban noveno y décimo semestre, respectivamente, determinando así una homogeneidad de la población, al mismo tiempo que refleja que eran estudiantes próximos a graduarse, por lo que se presume una concepción más clara del potencial de usar las TIC en su futuro profesional. Entre los criterios de inclusión de la muestra se encontraba: estar en el último año de su programa profesional, pertenecer a un programa de ingeniería, encontrarse matriculados como estudiantes activos y haber cursado al menos 2 asignaturas medidas por las TIC en sus carreras. Como criterios de exclusión se tenían: tener otro título universitario o profesión, ser becarios o monitores académicos en alguna asignatura mediada por TIC y encontrarse en proceso de cambio de carrera o pasantía en la universidad.

\section{Hipótesis}

Se formularon un total de 12 hipótesis, las cuales fueron agrupadas por componentes y se muestran en la Tabla 1. Como primer aspecto se tiene la autoeficacia, cuyo concepto cubre la autoconfianza de los estudiantes y sus expectativas para el rendimiento futuro, donde las experiencias de aprendizaje de éstos son cruciales para el desarrollo de creencias de autoeficacia, que pueden afectar sus logros (Hatlevik et al., 2018). Estudios como los de Rohatgi et al. (2016), confirman la existencia de diferentes formas de autoeficacia de las TIC, por lo tanto, es importante examinar esta distinción al elaborar el concepto de autoeficacia de las TIC.

Tabla 1: Hipótesis por componentes.

\begin{tabular}{|c|c|c|}
\hline Componentes & \multicolumn{2}{|c|}{ Hipótesis } \\
\hline Autoeficacia & $\begin{array}{l}\text { H1: La actitud percibida del uso de TIC como } \\
\text { soporte de la formación profesional } \\
\text { incrementa la percepción de autoeficacia }\end{array}$ & $\begin{array}{l}\text { H8: El reconocimiento de la importancia del uso } \\
\text { de software como soporte en la formación de } \\
\text { estudiantes de ingeniería favorece la percepción } \\
\text { de autoeficacia }\end{array}$ \\
\hline \multirow[t]{3}{*}{ Uso de TIC } & $\begin{array}{l}\text { H3: La mayoría de los estudiantes percibe } \\
\text { una utilidad positiva del uso de las TIC en la } \\
\text { educación }\end{array}$ & $\begin{array}{l}\text { H7: Las habilidades de los estudiantes en el uso } \\
\text { de herramientas institucionales contribuyen a la } \\
\text { utilidad de uso percibida de TIC }\end{array}$ \\
\hline & $\begin{array}{l}\text { H10: Reconocer la importancia del uso de } \\
\text { software como soporte en la formación de } \\
\text { estudiantes de ingeniería contribuye a la } \\
\text { utilidad de uso percibido de las TIC }\end{array}$ & $\begin{array}{l}\text { H11: La utilidad de uso percibida de las TIC } \\
\text { contribuye a la intención de uso de TIC como } \\
\text { soporte para la formación profesional en } \\
\text { estudiantes de ingeniería }\end{array}$ \\
\hline & \multicolumn{2}{|c|}{ H12: La preparación del estudiante ayuda a la utilidad de uso percibido de las TIC } \\
\hline \multirow[t]{2}{*}{$\begin{array}{l}\text { Empoderamiento } \\
\text { de las TIC }\end{array}$} & $\begin{array}{l}\text { H4: El control de la conducta frente al uso de } \\
\text { TIC por parte de los estudiantes de ingeniería } \\
\text { contribuye a la importancia del uso de } \\
\text { software como soporte en la formación de los } \\
\text { estudiantes }\end{array}$ & $\begin{array}{l}\text { H5: La facilidad de uso percibida de las TIC } \\
\text { como soporte en la formación profesional aporta } \\
\text { a la importancia del uso de software como } \\
\text { soporte en la formación de estudiantes de } \\
\text { ingeniería }\end{array}$ \\
\hline & \multicolumn{2}{|c|}{$\begin{array}{l}\text { H6: Las habilidades de los estudiantes en el uso de herramientas institucionales favorece } \\
\text { positivamente la intención de uso de TIC como soporte para la formación profesional }\end{array}$} \\
\hline Uso de LMS & $\begin{array}{l}\text { H2: La actitud percibida del uso de TIC como } \\
\text { soporte de la formación profesional contribuye } \\
\text { a la preparación del estudiante }\end{array}$ & $\begin{array}{l}\text { H9: Reconocer la importancia del uso de } \\
\text { software como soporte en la formación de } \\
\text { estudiantes de ingeniería mejora su preparación }\end{array}$ \\
\hline
\end{tabular}

Según los estudios de Vega, Patino y Galindo (2018), los estudiantes universitarios afirman que el uso de las TIC son un elemento importante en su formación académica, quienes aseguran que el uso de estas herramientas ayudan a su proceso de aprendizaje; así mismo, consideran el uso de éstas como un soporte para completar su conocimiento formativo. Sin embargo, existen diferencias significativas en las áreas de conocimiento, incluso el acceso y utilidad de estas tecnologías, no son uniformes entre países; además, el uso de estas tecnologías por parte de los universitarios depende de factores que van más allá de la disponibilidad (Gasaymeh, 2018). Desde el punto de vista profesional, el empoderamiento de las TIC, no sólo es afectada por la capacidad de los estudiantes para competir en el mercado laboral, sino que también afecta sus habilidades para aprender a aprender (learn-to-learn); la calidad, tipos de trabajos y las interacciones internacionales que obtienen; los salarios que reciben y el estatus que obtienen (Umunadi, 2011).

Gracias a los avances científicos, ha aumento la accesibilidad y ha disminuido el costo de diferentes artefactos y dispositivos tecnológicos como tabletas y computadores; este proceso a su vez ha favorecido la integración e implementación de estos dispositivos y artefactos tecnológicos al contexto pedagógico, que por efecto han promovido y favorecido la reforma de muchos sistemas educativos en el mundo ( $\mathrm{Li}, 2010)$. Para ello, las 
instituciones de educación superior tienen un papel clave en la promoción del conocimiento y la innovación, donde incorporar la enseñanza y uso de las TIC dentro de la formación, como un tema de capacitación cruzada, es un desafío, pero necesario en la preparación de los estudiantes para el éxito en el mercado profesional (Picatoste et al., 2018).

En el caso de la población de estudio, se hace énfasis en el uso de LMS (Learning Management System); dado esto, el e-learning o aprendizaje virtual, es una tecnología educativa moderna para facilitar el proceso de aprendizaje, con la ayuda de aplicaciones de software y entorno de aprendizaje virtual (Pham y Huynh, 2017). Sus ventajas son reconocidas en todo el mundo, pero su impacto en los logros de aprendizaje y el conocimiento no está confirmada claramente (Soeparno y Muslim, 2018). Por otro lado, el sistema de aprendizaje virtual, a menudo usa muchos tipos de medios para enviar texto, audio, imágenes y videos al aprendiz, un sistema comúnmente utilizado es el conocido como LMS, ya mencionado, como es el caso de la plataforma Moodle (crucial en esta investigación), los cuales pueden ayudar a crear y ofrecer algunos cursos, así como proporcionar la capacidad de simplificar las lecciones (Pham y Huynh, 2017).

\section{Procedimiento}

Se construyó inicialmente un instrumento, el cual fue formulado a partir de los principales hallazgos de la revisión preliminar de literatura, identificando variables susceptibles de medición acorde a las dimensiones de interés propuestas para la investigación, seguido a ello, el instrumento fue sometido a revisión por parte de investigadores con experiencia en el campo de interés del estudio. Este, facilitó la obtención de información acerca del nivel de acuerdo o desacuerdo que manifiestan los estudiantes de ingeniería de la Universidad Nacional frente a la necesidad de implementar las tecnologías de la información y las comunicaciones (TIC) como parte del proceso de aprendizaje; contando con un total de 23 ítems en escala de Likert, en un rango de respuesta de 1 a 5; así entonces, la representación categórica de la escala significa que: 1 = totalmente en desacuerdo; 2 = en desacuerdo; 3 = ni de acuerdo ni en desacuerdo; 4 = de acuerdo, y $5=$ totalmente de acuerdo. Las preguntas realizadas se agrupan en 4 dimensiones y se tienen en cuenta diferentes opciones de pregunta para evaluar cada dimensión, ya que estas no pueden ser medidas desde un solo criterio.

Las dimensiones mencionadas se detallan a continuación: la primera es la frecuencia del uso, esta implica aspectos como la práctica, permanencia, grado de conocimiento de las herramientas informáticas con las que cuenta la universidad y uso de estas. La segunda, es la importancia percibida de las TIC, la cual evalúa el nivel de relevancia que los estudiantes encuentran frente a la incorporación de las herramientas tecnológicas en su proceso de aprendizaje. La tercera, es la favorabilidad percibida, que mide la decisión de querer utilizar las tecnologías de la información y, a su vez, los dispositivos tecnológicos en su formación como ingenieros; y, por último, en la cuarta dimensión se encuentra la percepción de acceso desde la universidad, con la cual se buscó explorar cómo los estudiantes de ingeniería perciben el nivel de acceso a estas herramientas.

\section{Análisis de datos}

Los análisis estadísticos se realizaron utilizando el programa estadístico SPSS versión 22, para el análisis factorial exploratorio y confirmatorio. Inicialmente, se realizó el análisis factorial exploratorio (abreviado en el texto con las siglas AFE), definido como una técnica de reducción de datos basada en la búsqueda de patrones de correlaciones. El procedimiento realizado supuso analizar cada conjunto de variables de acuerdo a lo siguiente: en primer lugar, se aplicó a todo el grupo de variables el método de estimación por Máxima Verosimilitud, teniendo en cuenta que el conjunto de datos cumple con los requisitos mínimos para aplicar este método de estimación, ya que, se utiliza una muestra de 115 estudiantes de ingeniería; la cual es lo suficientemente grande para la estimación de los parámetros; por otro lado, las variables se distribuyen multi normalmente (Reise et al., 2000).

Finalmente, se usó el método de consistencia interna por medio del coeficiente de Cronbach (Cronbach, 1951), el cual refleja el grado en el que los ítems covarían Seguido de esto, se plantea un modelo en el que se busca validar 12 hipótesis, de acuerdo con los factores obtenidos después de efectuar el Análisis Factorial Exploratorio. Además, se corroboró la estructura factorial de cargas estandarizadas obtenidas para cada variable a través de la utilización del Análisis Factorial Confirmatorio.

\section{RESULTADOS}

Para clarificar la estructura factorial se realizó una rotación de ejes, a través del método Varimax (método de rotación ortogonal que minimiza el número de variables que tienen saturaciones altas en cada factor) y se obtuvo que las 23 variables analizadas se agrupan en nueve constructos, tal y como se señala en la Tabla 2. 
Tabla 2: Constructos encontrados de acuerdo con variables

\begin{tabular}{|c|c|}
\hline Constructo & Definición \\
\hline $\begin{array}{l}\text { Constructo } 1 \text { (Actitud percibida del uso de TIC como } \\
\text { soporte de la formación profesional - AP) }\end{array}$ & $\begin{array}{l}\text { Este Constructo está asociado a las variables relacionadas } \\
\text { con la importancia que tiene para el estudiante, el uso del } \\
\text { internet, las tabletas electrónicas y los dispositivos móviles } \\
\text { en los procesos de enseñanza y aprendizaje. }\end{array}$ \\
\hline $\begin{array}{l}\text { Constructo } 2 \text { (Control de la conducta frente al uso de TIC } \\
\text { por parte de los estudiantes de ingeniería - CC) }\end{array}$ & $\begin{array}{l}\text { Las variables agrupadas a este factor corresponden a } \\
\text { aquellas que evalúan el nivel de necesidad manifestado por } \\
\text { los estudiantes de ingeniería frente al uso de las TIC en su } \\
\text { proceso de aprendizaje. }\end{array}$ \\
\hline $\begin{array}{l}\text { Constructo } 3 \text { (Facilidad de uso percibida de las TIC } \\
\text { como soporte en la formación profesional - FP) }\end{array}$ & $\begin{array}{l}\text { Este constructo está asociado a las variables relacionadas } \\
\text { con la actitud favorable que los estudiantes consideran que } \\
\text { tendría el uso de redes sociales (Facebook, Twitter, } \\
\text { Messenger) como facilitadores del aprendizaje y qué tan } \\
\text { satisfechos están con las herramientas informáticas } \\
\text { utilizadas por sus docentes en las clases. }\end{array}$ \\
\hline $\begin{array}{l}\text { Constructo } 4 \text { (Habilidades del estudiante en el uso de } \\
\text { herramientas institucionales - HE) }\end{array}$ & $\begin{array}{l}\text { Las variables agrupadas a este factor corresponden a } \\
\text { aquellas que evalúan la frecuencia con la que los } \\
\text { estudiantes utilizan el correo institucional y la plataforma } \\
\text { Moodle que tiene actualmente la Universidad Nacional. }\end{array}$ \\
\hline $\begin{array}{l}\text { Constructo } 5 \text { (Importancia del uso de software como } \\
\text { soporte en la formación de estudiantes de ingeniería - } \\
\text { IS) }\end{array}$ & $\begin{array}{l}\text { Asociado a las variables relacionadas con la importancia } \\
\text { que los estudiantes de ingeniería le dan al uso de software } \\
\text { con licencia y software libre como soporte para su } \\
\text { formación. }\end{array}$ \\
\hline $\begin{array}{l}\text { Constructo } 6 \text { (Intención de uso de TIC como soporte } \\
\text { para la formación profesional en estudiantes de } \\
\text { ingeniería-IU) }\end{array}$ & $\begin{array}{l}\text { En este factor se encuentran las variables encargadas de } \\
\text { medir la usabilidad que los estudiantes le dan a las } \\
\text { herramientas tecnológicas e informáticas en el proceso de } \\
\text { enseñanza y aprendizaje universitario. }\end{array}$ \\
\hline Constructo 7 (Percepción de autoeficacia - PA) & $\begin{array}{l}\text { En este constructo se analizan las variables que indagan } \\
\text { sobre la frecuencia con la que los estudiantes de ingeniería } \\
\text { utilizan herramientas informáticas para realizar trabajos o } \\
\text { preparar exámenes. }\end{array}$ \\
\hline Constructo 8 (Preparación del estudiante - PE) & $\begin{array}{l}\text { Este constructo está asociado a las variables que } \\
\text { determinan la frecuencia con la que los estudiantes usan las } \\
\text { bases de datos académicas de las que dispone la } \\
\text { universidad y la plataforma Moodle como interfaz para } \\
\text { mejorar los procesos de aprendizaje y enseñanza. }\end{array}$ \\
\hline Constructo 9 (Utilidad de uso percibida de TIC - UP): & $\begin{array}{l}\text { Este constructo está conformado por dos ítems } \\
\text { relacionados con las bondades que experimentan los } \\
\text { estudiantes de ingeniería frente al uso de las herramientas } \\
\text { tecnológicas en sus procesos de aprendizaje, enmarcados } \\
\text { en dos características principales: la eficiencia y la } \\
\text { capacidad de resolver problemas. }\end{array}$ \\
\hline
\end{tabular}

En esta primera fase del análisis, se logró constatar que los datos recolectados en la investigación no parecen mostrar información redundante, ya que, las cargas factoriales de las variables observables en su mayoría fueron superiores a 0,6 (Bagozzi y Yi, 1988), logrando que el promedio de cargas factoriales para cada constructo pueda obtener un valor superior a 0,7 (Hair et al., 2010). Posterior a este análisis, en la Figura 1 se presenta el modelo de hipótesis a validar. Una vez efectuado el análisis factorial exploratorio (AFE), se realizó el análisis factorial confirmatorio (AFC), dado que, una práctica habitual para obtener evidencia de validez de modelos teóricos basados en los resultados del AFE, suele ser utilizar el AFC para demostrar la validez de la estructura factorial previamente obtenida, y, por consiguiente, la validez de las deducciones teóricas inferidas del mismo (Pérez et al., 2000). De acuerdo con lo anterior, el procedimiento que se llevó a cabo consistió en la validación de las escalas en las que se midió la información y la fiabilidad del instrumento de medida utilizado. 


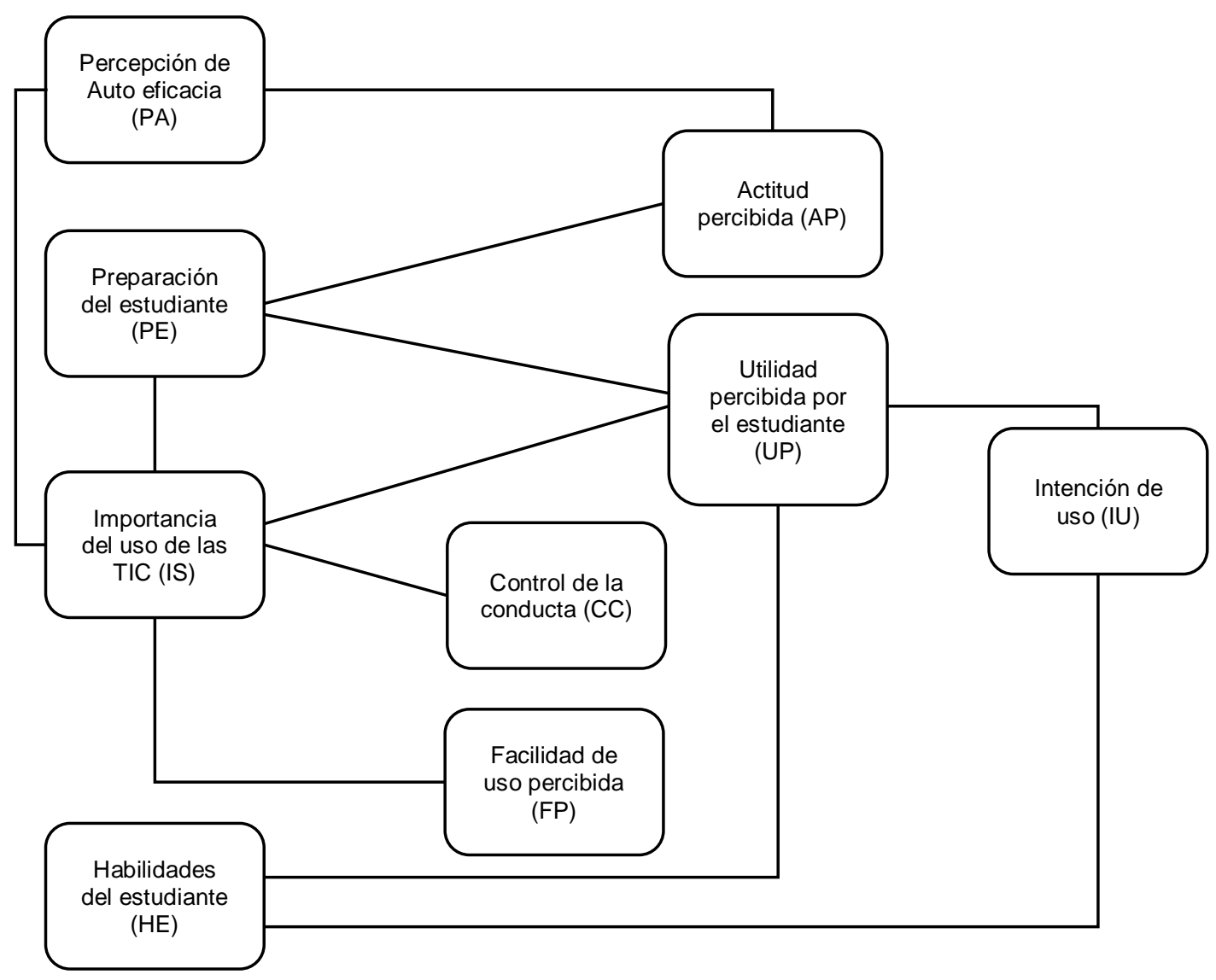

Fig. 1: Modelo propuesto para valorar la intención de uso de TIC como soporte para la formación profesional en estudiantes de ingeniería.

\section{Validez Convergente}

Como se mencionó anteriormente, la validez de las escalas de medida utilizadas junto con cada uno de los constructos y el instrumento en general se comprobó a través del método estadístico análisis factorial confirmatorio, dicho análisis representa un conjunto de diversos procedimientos técnicos para el estudio de la relación de interdependencia entre un conjunto de variables con la finalidad de agruparlas en función de la "variabilidad compartida"; descubrir las estructuras subyacentes (factores), dimensiones o conceptos latentes, cumpliendo la finalidad de resumir y reducir los datos (Pérez y Medrano, 2010).

Frente a lo anterior, se debe tener en cuenta que, la fiabilidad del modelo se valora en dos niveles, por un lado la fiabilidad de los ítems observables; y por otro lado se valora la fiabilidad de los constructos (Calvo et al., 2013). Y para determinar su impacto sobre el modelo, algunos autores han establecido que, una fiabilidad superior a 0.6 es considerada como evidencia de que el modelo es fiable (Bagozzi y Yi, 1988). Así mismo, la fiabilidad de los constructos se refiere al grado con que una variable observable refleja un factor, considerándose aceptable un valor superior a 0.7 (Hair et al., 2010). Se tiene entonces que, la validez convergente evalúa el grado en que la medida de los ítems que recogen un mismo concepto están correlacionadas (Calvo et al., 2013). En el presente estudio no fue necesario eliminar ningún indicador, dado que, su carga factorial estandarizada cumplen con los criterios de evaluación establecidos por los autores citados previamente, tal y como se muestra en la Tabla 3.

Tabla 3: Validez convergente de cargas factoriales estandarizadas

\begin{tabular}{|l|c|c|c|}
\hline \multirow{2}{*}{ Constructo } & Ítem & $\begin{array}{c}\text { Cargas factoriales } \\
\text { estandarizadas }\end{array}$ & $\begin{array}{c}\text { Promedio de cargas factoriales } \\
\text { estandarizadas }\end{array}$ \\
\hline \multirow{4}{*}{ Actitud percibida del uso de TIC } & AP1 & 0.531 & \multirow{2}{*}{0.7} \\
\cline { 2 - 3 } & AP2 & 0.817 & \\
\cline { 2 - 3 } & AP3 & 0.830 & \multirow{2}{*}{0.8} \\
\hline \multirow{2}{*}{$\begin{array}{l}\text { Control de la conducta frente al uso de } \\
\text { TIC }\end{array}$} & CC1 & 0.822 & \\
\cline { 2 - 3 } & CC2 & 0.822 & \\
\hline
\end{tabular}


Tabla 3: continuación

\begin{tabular}{|c|c|c|c|}
\hline Constructo & Ítem & $\begin{array}{l}\text { Cargas factoriales } \\
\text { estandarizadas }\end{array}$ & $\begin{array}{c}\text { Promedio de cargas } \\
\text { factoriales estandarizadas }\end{array}$ \\
\hline \multirow{2}{*}{ Facilidad de uso percibida de las TIC } & FP1 & 0.752 & \multirow{2}{*}{0.8} \\
\hline & FP2 & 0.752 & \\
\hline \multirow{2}{*}{ Habilidades de los estudiantes } & $\mathrm{HE} 1$ & 0.812 & \multirow{2}{*}{0.8} \\
\hline & HE2 & 0.812 & \\
\hline \multirow{2}{*}{ Importancia del uso de software } & IS1 & 0.872 & \multirow{2}{*}{0.9} \\
\hline & IS2 & 0.872 & \\
\hline \multirow{6}{*}{$\begin{array}{l}\text { Intención de uso de TIC como soporte } \\
\text { para la formación profesional en } \\
\text { estudiantes de ingeniería }\end{array}$} & IU1 & 0.600 & \multirow{6}{*}{0.7} \\
\hline & IU2 & 0.668 & \\
\hline & IU3 & 0.565 & \\
\hline & IU4 & 0.771 & \\
\hline & IU5 & 0.738 & \\
\hline & IU6 & 0.746 & \\
\hline \multirow{2}{*}{ Percepción de autoeficacia } & PA1 & 0.781 & \multirow{2}{*}{0.8} \\
\hline & PA2 & 0.781 & \\
\hline \multirow{2}{*}{ Preparación del estudiante } & PE1 & 0.833 & \multirow{2}{*}{0.8} \\
\hline & PE2 & 0.833 & \\
\hline \multirow{2}{*}{ Utilidad de uso percibida de TIC } & UP1 & 0.820 & \multirow{2}{*}{0.8} \\
\hline & UP2 & 0.820 & \\
\hline
\end{tabular}

\section{Validez Discriminante}

La validez discriminante es uno de los criterios habituales para evaluar las escalas de medida de constructos latentes en ciencias sociales, en esta fase se afirma que, para que unas medidas sean válidas, las de un mismo constructo deben correlacionar altamente entre ellas, y esa correlación debe ser mayor de la que exista con respecto a las medidas propuestas para otro constructo distinto (Campbell y Fiske, 1959). El análisis de validez discriminante se ejecutó mediante la comprobación de que el intervalo de confianza en la estimación de la correlación entre cada par de factores no contuviera el valor 1 (Anderson y Gerbing, 1988). La Tabla 4 evidencia que todos los casos cumplen con dicho criterio y de esta forma se constata que, la validez del instrumento utilizado mide en alto grado aquello que pretendía medir.

Tabla 4: Validez discriminante del modelo de medida

\begin{tabular}{|c|c|c|c|c|c|c|c|c|c|}
\hline & AP & CC & FP & HE & IS & IU & PA & PE & UP \\
\hline AP & $\ldots$ & & & & & & & \\
\hline CC & {$[-0.301 ;-0.114]$} & $\ldots$ & & & & & & & \\
\hline FP & {$[-0.099 ; 0.354]$} & {$[0.005 ; 0.102]$} & $\ldots$ & & & & & & \\
\hline HE & {$[-0.061 ; 0.293]$} & {$[-0.086 ;-0.026]$} & {$[-0.033 ; 0.372]$} & $\ldots$ & & & & & \\
\hline IS & {$[-0.234 ; 0.187]$} & {$[-0.065 ; 0.383]$} & {$[-0.084 ; 0.270]$} & {$[-0.135 ; 0.332]$} & $\ldots$ & & & & \\
\hline IU & {$[-0.175 ; 0.254]$} & {$[-0.204 ; 0.229]$} & {$[-0.102 ; 0.323]$} & {$[0.022 ; 0.455]$} & {$[-0.154 ; 0.158]$} & $\ldots$ & & & \\
\hline PA & {$[0.099 ; 0.451]$} & {$[-0.204 ; 0.229]$} & {$[-0.199 ; 0.247]$} & {$[-0.033 ; 0.371]$} & {$[0.077 ; 0.470]$} & {$[-0.111 ; 0.307]$} & $\ldots$ & & \\
\hline PE & {$[-0.065 ; 0.291]$} & {$[0.075 ; 0.239]$} & {$[-0.193 ; 0.308]$} & {$[-0.133 ; 0.359]$} & {$[-0.033 ; 0.328]$} & {$[-0.014 ; 0.348]$} & {$[-0.008 ; 0.410]$} & $\ldots$ \\
\hline UP & {$[-0.063 ; 0.418]$} & {$[-0.112 ; 0.299]$} & {$[-0.072 ; 0.289]$} & {$[-0.116 ; 0.372]$} & {$[-0.012 ; 0.525]$} & {$[-0.228 ; 0.199]$} & {$[-0.101 ; 0.365]$} & {$[-0.125 ; 0.232]$} & $\ldots$ \\
\hline
\end{tabular}


Por otro lado, la fiabilidad de la consistencia interna del instrumento se estimó por medio del alfa de Cronbach, en vista de que es una herramienta en la que se asume que los ítems (escala tipo Likert) miden a un mismo constructo y están altamente correlacionados. Y en lo que respecta a su interpretación, se tiene que, si el valor del alfa de Cronbach es más cercano a 1, es mayor la consistencia interna de los ítems, ya que, la prueba alcanza valores positivos comprendidos entre 0 y 1 , donde 0 indica ausencia total de consistencia interna, y 1 , la redundancia total entre los ítems. Como se observa en la Tabla 5, el instrumento de medida parece tener una buena fiabilidad de la escala de medida.

Tabla 5: Índice de fiabilidad - Alfa de Cronbach

\begin{tabular}{|c|c|}
\hline Constructo & Alfa de Cronbach \\
\hline AP & 0.760 \\
\hline CC & 0.698 \\
\hline FP & 0.735 \\
\hline HE & 0.798 \\
\hline IS & 0.875 \\
\hline IU & 0.831 \\
\hline PA & 0.765 \\
\hline PE & 0.840 \\
\hline UP & 0.817 \\
\hline
\end{tabular}

\section{Contraste de hipótesis}

Se procedió a realizar la estimación del modelo estructural propuesto para evaluar sobre qué base determinan el uso de las TIC los estudiantes de ingeniería de la Universidad Nacional de Colombia, Sede Medellín, en donde se recogen las diversas hipótesis planteadas y se mide su grado de asociación por medio del estadístico V de Cramer, el cual arroja el grado de asociación entre las variables tenidas en cuenta para la formulación de hipótesis, tomando valores entre 0 y 1, así las cosas, cuando alcanza los valores extremos existe concordancia y los valores próximos a 0 indican ausencia de asociación (Fierro, 2010). En la Tabla 6 se encuentran los valores obtenidos para el estadístico de asociación analizado y el modelo utilizado.

Tabla 6: Validación de hipótesis, grado de asociación de factores

\begin{tabular}{|l|c|c|}
\hline & Hipótesis & V de Cramer \\
\hline $\mathrm{H} 1$ & $\mathrm{AP} \rightarrow \mathrm{PA}$ & 0.232 \\
\hline $\mathrm{H} 2$ & $\mathrm{AP} \rightarrow \mathrm{PE}$ & 0.233 \\
\hline $\mathrm{H} 3$ & $\mathrm{AP} \rightarrow \mathrm{UP}$ & 0.232 \\
\hline $\mathrm{H} 4$ & $\mathrm{CC} \rightarrow \mathrm{IS}$ & 0.282 \\
\hline $\mathrm{H} 5$ & $\mathrm{FP} \rightarrow \mathrm{IS}$ & 0.271 \\
\hline $\mathrm{H} 6$ & $\mathrm{HE} \rightarrow \mathrm{IU}$ & 0.301 \\
\hline $\mathrm{H} 7$ & $\mathrm{HE} \rightarrow \mathrm{UP}$ & 0.292 \\
\hline $\mathrm{H} 8$ & $\mathrm{IS} \rightarrow \mathrm{PA}$ & 0.329 \\
\hline $\mathrm{H} 9$ & $\mathrm{IS} \rightarrow \mathrm{PE}$ & 0.238 \\
\hline $\mathrm{H} 10$ & $\mathrm{IS} \rightarrow \mathrm{UP}$ & 0.428 \\
\hline $\mathrm{H} 11$ & $\mathrm{UP} \rightarrow \mathrm{IU}$ & 0.300 \\
\hline $\mathrm{H} 12$ & $\mathrm{PE} \rightarrow \mathrm{UP}$ & 0.247 \\
\hline
\end{tabular}

En la Figura 2, se presenta el respectivo modelo propuesto con el valor de asociación entre las variables. Éste permite validar las hipótesis generadas a partir del análisis exploratorio y del objetivo principal de este trabajo, enmarcado en indagar la relación, importancia y uso que tienen las TIC en el proceso de aprendizaje de los estudiantes de ingeniería administrativa e industrial de séptimo semestre en adelante de la Universidad Nacional de Colombia- Sede Medellín. 


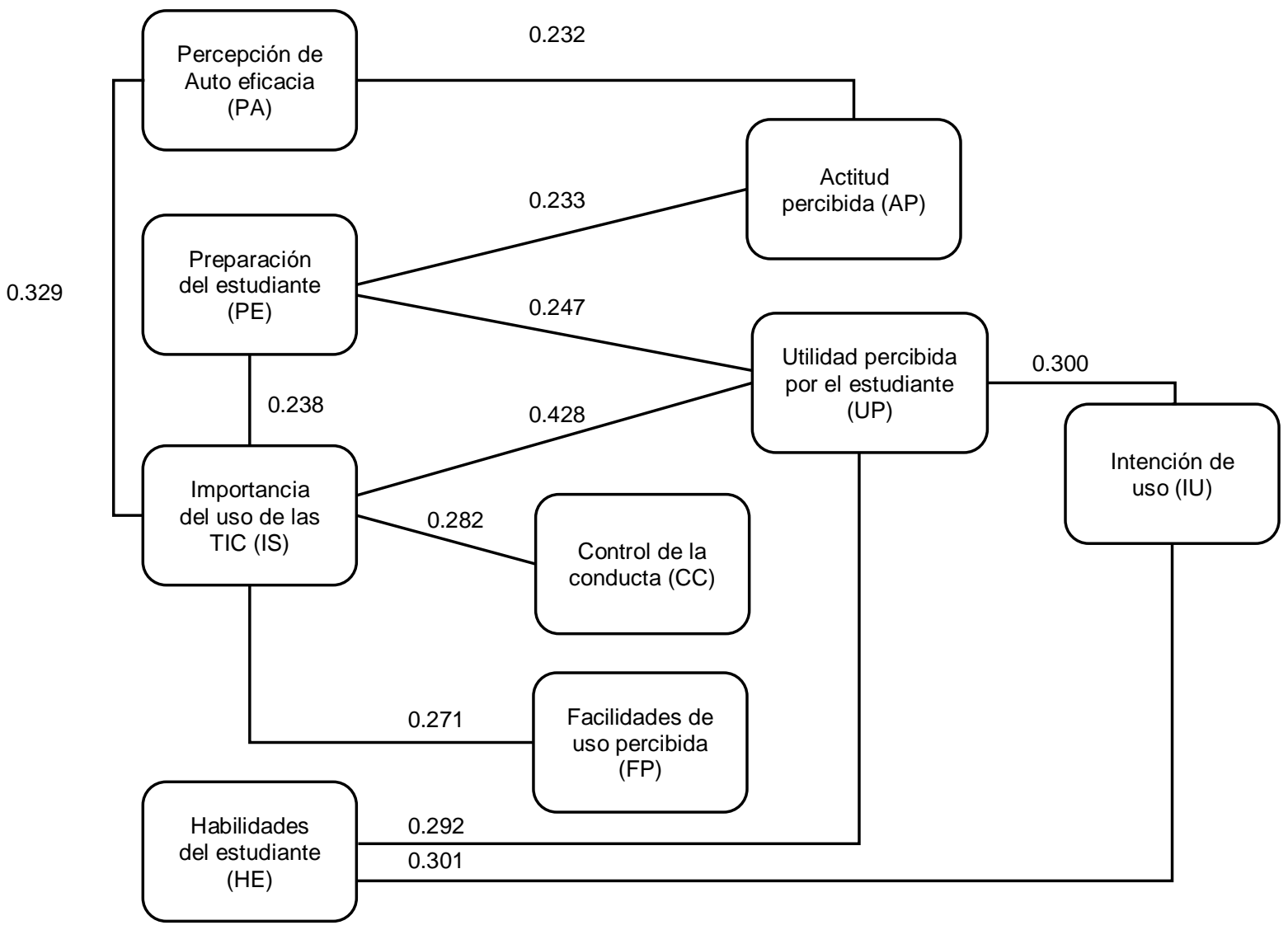

Fig. 2: Modelo propuesto, V de Cramer. Las líneas no direccionadas indican las hipótesis nulas que se validaron en la investigación.

\section{DISCUSIÓN}

Esta investigación busca estudiar los factores asociados a la intención de uso de las TIC en el aprendizaje de estudiantes de ingeniería, en ese sentido, en este estudio se asume como relación significativa a aquellas cuyos niveles de asociación, con coeficiente $\mathrm{V}$ de Cramer, sean igual o superior a 0.3; apoyado en Fierro (2010) quien afirma que en el marco de los estudios de las ciencias sociales, se puede interpretar el coeficiente $\mathrm{V}$ de Cramer de la siguiente manera: asociación baja $(0.0 \leq \mathrm{V}$ de Cramer $<0.2)$, asociación media $(0.2 \leq \mathrm{V}$ de Cramer $<0.3)$; y asociación alta $(0.3 \leq \mathrm{V}$ de Cramer $\leq 1.0)$.

La relación más significativa del modelo propuesto se dio entre el reconocimiento de la importancia del uso de software como soporte en la formación de estudiantes de ingeniería y la utilidad de uso percibida de TIC, representada por la hipótesis H10; con un grado de asociación de 0.428 , según coeficiente de V de Cramer. Este resultado indica que la percepción de importancia TIC es la mejor variable explicativa de la utilidad de uso percibida, que a su vez es uno de los constructos más influyentes en la intención de uso.

En general, los estudios sobre la percepción de utilidad de las TIC en el aprendizaje resaltan la importancia del uso de estas herramientas; sin embargo, no se encontró evidencia de investigaciones enfocadas en analizar la relación entre percepción de importancia y percepción de utilidad de uso. Una explicación por la cual se presenta este comportamiento es que estos estudios están basados en el Modelo de Aceptación Tecnológica (TAM) propuesto por Davis (1989) o en adaptaciones del mismo, modelo que no contempla la percepción de importancia como factor de análisis. Lo cual se corrobora con estudios como los de Gasaymeh (2018); Kwok y Yang (2017); Park (2009); quienes estudiaron diferentes factores que inciden en el uso de las TIC, basados en el modelo TAM, sin considerar la percepción de importancia.

Otra relación importante encontrada en el modelo propuesto es la presentada entre el reconocimiento de importancia del uso de las TIC y la percepción de autoeficacia, representada por la hipótesis H8; el resultado muestra un grado de asociación de 0.329, dado por el coeficiente V de Cramer. Ching-Ter et al. (2017) afirman que la percepción de autoeficacia es un factor que debe ser tenido en cuenta en el análisis de intención de uso de TIC, dado que afecta positivamente la percepción de facilidad de uso y este último a la intención de uso. Así mismo, los estudios de Rohatgi et al. (2016), quienes indagaron sobre la influencia de la percepción de autoeficacia en la intención de uso; plantearon que, desde el punto de vista motivacional, la percepción de 
autoeficacia es el factor más importante que incide positivamente en la intención de uso de las TIC en la educación. Pese a lo anterior, no se encontró evidencia de estudios que relacionen directamente la percepción de autoeficacia con el reconocimiento de la importancia del uso de las TIC. Una explicación a esto es el hecho de que el factor autoeficacia es normalmente analizado por su incidencia directa en la intención de uso de las TIC (como variable de salida), y no desde su relación con otros constructos.

La siguiente relación significativa está representada por la hipótesis H6, la cual hace referencia a que las habilidades de los estudiantes en el uso de herramientas institucionales favorecen positivamente la intención de uso de TIC como soporte para la formación profesional; con un coeficiente V de Cramer de 0.301. Verhoeven et al. (2010) y Callum y Jeffrey (2013) encontraron resultados similares, donde plantearon que las habilidades en el uso de TIC, son significativas e importantes para explicar la intención de su uso por parte de los estudiantes; sin embargo, en esos estudios, este no era el constructo más significativo, en ello pudo haber incidido distintas variables; por un lado las características demográficas de la población de estudio, dado que en la muestra analizada en la presente investigación, los estudiantes desarrollan habilidades de uso de las TIC en la educación desde tempranos semestres. Por otro lado, se tiene que las herramientas TIC particulares utilizadas por estudiantes de distintas universidades y regiones, pueden presentar diferencias entre sí; ocasionando variaciones en los resultados de las investigaciones.

La cuarta relación significativa es la entablada entre la percepción de utilidad percibida del uso de las TIC en la educación y la intención de uso de las mismas, la cual está representada por la hipótesis H11; donde se obtuvo un grado de asociación de 0.300 , según coeficiente $\mathrm{V}$ de Cramer. Este resultado se comporta de acuerdo a lo esperado y coherente con otras investigaciones. Kwok y Yang (2017) plantearon, entre sus hallazgos, que la utilidad percibida del uso de las TIC en la educación es una de las variables que mejor explica la intención de uso de estas tecnologías. Además, la percepción de utilidad es uno de los factores contemplados en el modelo TAM de Davis (1989) y sus adaptaciones, sobre lo que se basan diferentes estudios sobre la intención de uso TIC en la educación.

Se tiene que los resultados de esta investigación han sido coherentes con los arrojados por otras investigaciones; no obstante, hay factores cuyo comportamiento varía de un estudio a otro. Esta conducta puede ser explicada por el hecho de que los contextos económicos son distintos, en el sentido de que algunos estudios son realizados en países desarrollados y otros en regiones emergentes o en desarrollo; afectando así algunos factores de incidencia en el uso de las TIC en la educación. Lo cual es corroborado con Gasaymeh (2018), quien afirma que existen diferencias en las áreas de conocimiento, asegurando que el acceso y utilidad de las TIC, no es percibido uniformemente entre países, incluso puede variar dentro de un país.

El uso de las TIC en entornos de enseñanza y aprendizaje han ido en incremento en los últimos años, como es el caso de Colombia que, en el contexto de países latinoamericanos, ha implementado diferentes estrategias para una correcta incorporación de estas tecnologías en contextos educativos. Atendiendo al propósito de esta investigación, que buscó identificar los factores que influyen en la intención de uso de softwares y herramientas TIC en la educación, finalmente se encontraron 4 relaciones significativas entre constructos, representadas por las hipótesis $\mathrm{H} 10, \mathrm{H} 8, \mathrm{H} 6$ y H11. En ese sentido, los estudiantes expresan que la percepción de importancia del uso de las TIC es uno de los factores más influyentes, puesto que incide significativamente en la utilidad percibida y en la percepción de auto eficacia. Así mismo, se encontró que, las habilidades que poseen los estudiantes y la utilidad percibida, son factores que inciden significativamente en la intención de uso.

Si bien este estudio refuerza el conocimiento relacionado al uso de modelos de adopción tecnológica para el entendimiento de los factores que inciden en el uso de las TIC en la educación, se expresa que el uso de estos modelos, y es el caso de este estudio, no se precisa la interdependencia que pueda existir entre los factores analizados. Adicionalmente, la muestra poblacional analizada corresponde a estudiantes de nivel universitario, con lo que la investigación no permite discernir en qué medida las percepciones de los encuestados son propias del nivel de formación universitario o corresponden a un conjunto de apreciaciones construidas desde niveles inferiores de formación. Así mismo, los resultados del estudio no son concluyentes en el sentido de que no afirman que los hallazgos obtenidos expliquen el comportamiento de estudiantes de otras ingenierías u otras universidades; con lo que se recomienda sea punto de partida para el desarrollo de futuras investigaciones.

En el contexto de países de economías en desarrollo, particularmente en Colombia, se tienen múltiples retos en cuanto a cobertura de la educación, aplicación de nuevas metodologías educativas, entre otras, que pueden ser mitigadas mediante la integración de Tecnologías de la Información y la Comunicación a los entornos de enseñanza-aprendizaje; en ese sentido, los resultados de estudio arrojan evidencia a favor de que las relaciones significativas entre los diferentes constructos varían dependiendo de la región y de las dinámicas socioeconómicas propias de cada zona. 


\section{CONCLUSIONES}

De acuerdo con el trabajo presentado y a los resultados obtenidos, se pueden plantear las siguientes conclusiones principales:

1.- La importancia del uso de las TIC en estudiantes universitarios se percibe como significativa y útil de acuerdo con el contexto al que ellos pertenecen, volviéndose éstas en herramientas trascendentales para mejorar el perfil y el desempeño a nivel personal y profesional.

2.- La relevancia en el uso de las TIC está medida por la utilidad percibida del estudiante en cuanto a la determinación a usar herramientas tecnológicas y comunicativas, intención marcada en los constructos señalados en el modelo propuesto.

3.- Finalmente, se obtiene que el desarrollo de las habilidades de los estudiantes para el uso de herramientas institucionales contribuye a su formación profesional, pues su intención de uso está favorecida por la autoeficacia percibida que se obtiene gracias a su trayectoria y dinámicas socioeconómicas según el contexto con el que se revise.

\section{REFERENCIAS}

Anderson, J. C., y Gerbing, D. W., Structural Equation Modeling in Practice: A Review and Recommended Two-Step Approach. https://doi.org/10.1037/0033-2909.103.3.411, Psychological Bulletin, 103(3), 411-423 (1988).

Bagozzi, R. P., y Yi, T., On the Evaluation of Structural Equation Models. Journal of the Academy of Marketing Science, 16(1), 74-94 (1988).

Bidarian, S., Bidarian, S., y Davoudi, A. M., A Model for application of ICT in the process of teaching and learning. https://doi.org/10.1016/j.sbspro.2011.11.336, Procedia - Social and Behavioral Sciences, 29(2010), 1032-1041 (2011).

Callum, K. M., y Jeffrey, L., The influence of students' ICT skills and their adoption of mobile learning. Australasian Journal of Educational Technology, 29(3), 303-314 (2013).

Calvo Porral, C., Martínez Fernández, V., y Juanatey Boga, S., Análisis de dos modelos de ecuaciones estructurales alternativos para medir la intencion de compra. Investigacion Operacional, 34(3), 230-243 (2013).

Campbell, D. T., y Fiske, D. W., Convergent and discriminant validation by the multitrait-multimethod matrix. https://doi.org/10.1037/h0046016, Psychological Bulletin, 56(2), 81-105 (1959).

Ching-Ter, C., Hajiyev, J., y Su, C.-R., Examining the students' behavioral intention to use e-learning in Azerbaijan? The General Extended Technology Acceptance Model for E-learning approach. https://doi.org/10.1016/j.compedu.2017.04.010, Computers and Education, 111, 128-143 (2017).

Cobo, C., El concepto de tecnologías de la información. Benchmarking sobre las definiciones de las TIC en la sociedad del conocimiento. Zer, 14(27), 295-318 (2009).

Cronbach, L. J., Coefficient alpha and the internal structure of tests. https://doi.org/10.1007/BF02310555, Psychometrika, 16(3), 297-334. (1951).

Davis, F. D., Perceived usefulness, perceived ease of use, and user acceptance of information technology. https://doi.org/10.2307/249008, MIS Quarterly: Management Information Systems, 13(3), 319-339 (1989).

Fierro, J., Análisis estadístico univariado, bivariado y variables control (pp. 1-16). pp. 1-16 (2010).

Gasaymeh, A., A Study of Undergraduate Students' Use of Information and Communication Technology (ICT) and the Factors Affecting their Use: A Developing Country Perspective. https://doi.org/10.29333/ejmste/85118, Eurasia Journal of Mathematics, Science and Technology Education, 14(5), 1731-1746 (2018).

Guerin, C., Jayatilaka, A., y otros tres autores. Research degrees in Information and Communication Technology (ICT): Why so few doctoral students?. https://doi.org/10.1080/0309877X.2016.1159290, Journal of Further and Higher Education, 41(5), 625-641 (2017).

Hair, J. F., Black, W. C., y otros dos autores. Multivariate data analysis: A global perspective, Pearson Education, United Kingdom (2010).

Hamidi, F., Meshkat, M., y otros dos autores. Information technology in education. https://doi.org/10.1016/j.procs.2010.12.062, Procedia Computer Science, 3, 369-373 (2011).

Hatlevik, O. E., Throndsen, I., y otros dos autores. Students' ICT self-efficacy and computer and information literacy: Determinants and relationships. https://doi.org/10.1016/j.compedu.2017.11.011, Computers and Education, 118(November 2017), 107-119 (2018)

Kwok, D., y Yang, S., Evaluating the intention to use ICT collaborative tools in a social constructivist environment. https://doi.org/10.1186/s41239-017-0070-1, International Journal of Educational Technology in Higher Education, 14(1), $1-14$ (2017). 
Li, S. C., Social capital, empowerment and educational change: a scenario of permeation of one-to-one technology in school. https://doi.org/10.1111/j.1365-2729.2010.00350.x, Journal of Computer Assisted Learning, 26(4), 284-295 (2010).

Maleki, H., Majidi, A., y otros tres autores. Effect of Applying Informant on and Communication Technology (ICT) on Learning Level and Information Literacy of Students. https://doi.org/10.1016/j.sbspro.2012.06.530, Procedia - Social and Behavioral Sciences, 46, 5862-5867 (2012).

Martínez, O., y otros cuatro autores, Estrategias pedagógicas aplicadas a la educación con mediación virtual para la generación del conocimiento global. https://doi.org/10.4067/S0718-50062018000500011, Formación Universitaria, 11(5), 11-18, (2018).

Meléndez, C. F., y Flores, L. D., Variación de la autonomía en el aprendizaje, en función de la gestión del conocimiento, para disminuir en los alumnos los efectos del aislamiento. https://doi.org/10.6018/red/50/5, Revista de Educación a Distancia, 54(7), 1-15 (2017).

Ornela, M., y otros tres autores, Autoeficacia percibida en la conducta académica de estudiantes universitarias, doi: https://doi.org/10.4067/S0718-50062012000200003, Formación Universitaria, 5(2), 17-26, (2012).

Park, S. Y., An Analysis of the Technology Acceptance Model in Understanding University Students' Behavioral Intention to Use e-Learning. Educational Technology \& Society, 12(3), 150-162 (2009).

Pérez, E., y Medrano, L., Análisis factorial exploratorio: Bases conceptuales y metodológicas. Revista Argentina de Ciencias Del Comportamiento, 2(1), 58-66 (2010).

Pérez, J. A., Chacón, S., y Moreno, R., Validez de constructo: El uso de análisis factorial exploratorio-confirmatorio para obtener evidencias de validez. Psicothema, 12(SUPPL. 2), 442-446 (2000).

Pham, Q. T., y Huynh, M. C., Impact factor on learning achievement and knowledge transfer of students through e-learning system at Bach Khoa University, Vietnam. https://doi.org/10.1109/ICCNI.2017.8123796, 2017 International Conference on Computing Networking and Informatics (ICCNI), 1-6 (2017).

Picatoste, J., Pérez Ortiz, L., y Ruesga Benito, S. M., A new educational pattern in response to new technologies and sustainable development. Enlightening ICT skills for youth employability in the European Union. https://doi.org/10.1016/j.tele.2017.09.014, Telematics and Informatics, 35(4), 1031-1038 (2018).

Reise, S. P., Waller, N. G., y Comrey, A. L., Factor analysis and scale revision. https://doi.org/10.1037//10403590.12.3.287, Psychological Assessment, 12(3), 287-297 (2000).

Rohatgi, A., Scherer, R., y Hatlevik, O. E., The role of ICT self-efficacy for students' ICT use and their achievement in a computer and information literacy test. https://doi.org/10.1016/j.compedu.2016.08.001, Computers and Education, 102, 103-116 (2016).

Soeparno Muslim, S., Effectiveness of E-Learning for Students Vocational High School Building Engineering Program. https://doi.org/10.1088/1757-899X/336/1/012039, IOP Conference Series: Materials Science and Engineering, 336(1), 06 (2018).

Umunadi, E. K., Perception of Technical Education Students on the Role of ICT in General Studies Programme (GSP) In University Education. https://doi.org/10.6007/ijarbss.v1i2.32, International Journal of Academic Research in Business and Social Sciences, 1(3), 17 (2011).

Verhoeven, J. C., Heerwegh, D., y De Wit, K., Information and communication technologies in the life of university freshmen: An analysis of change. https://doi.org/10.1016/j.compedu.2009.12.002, Computers and Education, 55(1), 5366 (2010). 